\title{
Observational evidence of CMEs interacting in the inner heliosphere as inferred from MHD simulations
}

\author{
N. Lugaz a,b,*, W. B. Manchester IV ${ }^{\text {a }}$, I. I. Roussev ${ }^{\text {b }}$, T. I. Gombosi ${ }^{\text {a }}$ \\ ${ }^{a}$ Center for Space Environment Modeling, University of Michigan, 2455 Hayward St., Ann Arbor, MI 48109, USA \\ ${ }^{\mathrm{b}}$ Institute for Astronomy, University of Hawaii, 2680 Woodlawn Dr., Honolulu, HI 96822, USA
}

Accepted 29 August 2007 doi:10.1016/j.jastp.2007.08.033

\begin{abstract}
The interaction of multiple Coronal Mass Ejections (CMEs) has been observed by LASCO coronagraphs and by nearEarth spacecraft, and it is thought to be an important cause of geo-effective storms, large Solar Energetic Particles events and intense Type II radio bursts. New and future missions such as STEREO, the LWS Sentinels, and the Solar Orbiter will provide additional observations of the interaction of multiple CMEs between the Sun and the Earth. We present the results of simulations of two and more CMEs interacting in the inner heliosphere performed with the Space Weather Modeling Framework (SWMF). Based on those simulations, we discuss the observational evidence of the interaction of multiple CMEs, both in situ and from coronagraphs. The clearest evidence of the interaction of the CMEs are the large temperature in the sheath, due to the shocks merging, and the brightness increase in coronagraphic images, associated with the interaction of the leading edges. The importance of having multiple satellites at different distances and angular positions from the Sun is also discussed.
\end{abstract}

Key words: MHD, shock wave, Sun, Coronal Mass Ejections

\section{Introduction}

Coronal Mass Ejections (CMEs) are the most extreme events occurring in our solar system, and their frequency highly depends on the phase of the solar cycle: from 6 a day near solar maximum to $0.5-$ 0.8 a day near solar minimum (Gopalswamy, 2004). The typical propagation time of a CME from the Sun to the Earth is 2-3 days. Therefore, near solar maximum, there is a high probability that multiple CMEs will interact on their way to Earth. Ejecta resulting from the interaction of multiple CMEs have been reported and studied by Burlaga et al. (2002);

\footnotetext{
* Corresponding author. Institute for Astronomy, University of Hawaii, 2680 Woodlawn Dr., Honolulu, HI 96822, USA Tel: +1 8089568534

Email address: nlugaz@umich.edu (N. Lugaz ).
}

Wang et al. (2003); Berdichevsky et al. (2003) and Farrugia \& Berdichevsky (2004), among others. Numerical investigations of multiple CMEs propagating and interacting, including the simpler case of the interaction between a CME and a forward shockwave, have been pioneered by Vandas et al. (1997) and recently reported by Odstrčil et al. (2003); Gonzalez-Esparza et al. (2004); Schmidt \& Cargill (2004); Lugaz et al. (2005, 2007) and Xiong et al. (2006).

Based on near-Earth in situ measurements only, the interaction region between the magnetic subclouds of a multiple-magnetic cloud event is among the only evidence that multiple CMEs interacted between the Sun and the Earth. This region is characterized by a lower magnetic field strength, a higher temperature, resulting in a larger plasma 


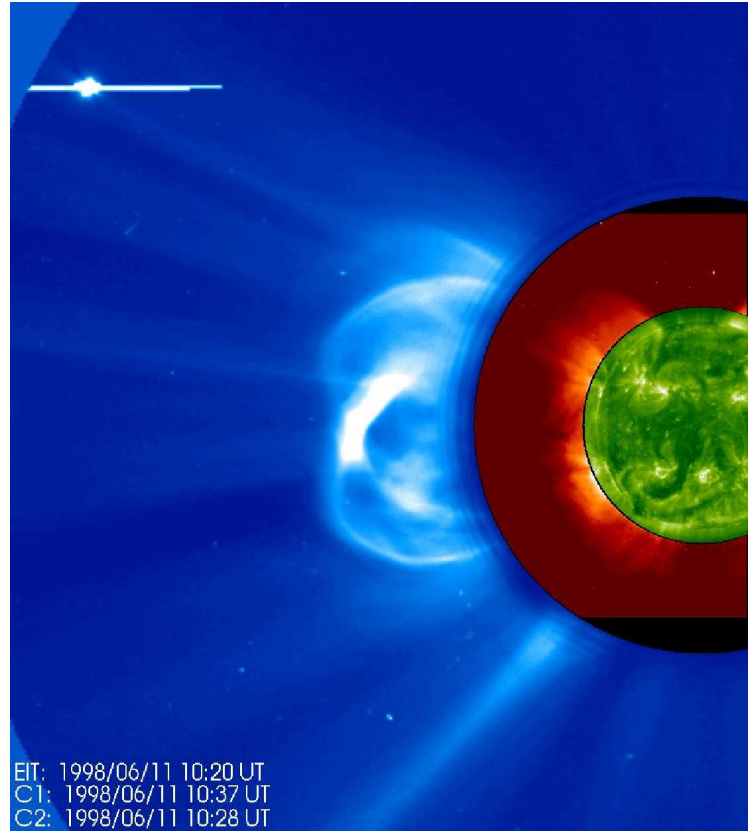

Fig. 1. Composite images (from SOHO/LASCO C1, C2 and EIT ) of two CMEs interacting in the solar corona on June 11, 1998. The two loops correspond to two sympathetic ejections.

$\beta$ (ratio of the thermal to the magnetic pressures) and it is associated with the reconnection between the two clouds (Wang et al., 2003). As noted by Burlaga et al. (2002), the speed profile of complex ejecta, although irregular, often shows variations of less than $100 \mathrm{~km} \mathrm{~s}^{-1}$ between the different ejecta. Thus, it can be hard to distinguish between an isolated CME and interacting CMEs, simply based on the speed profile of the events observed near-Earth.

The interaction of two CMEs near the Sun can sometimes be observed by the LASCO coronagraphs (e.g. Gopalswamy et al., 2001; Reiner et al., 2003). It can appear as CME "cannibalism" (Gopalswamy et al., 2001), where the faster ejection "swallows" the slower, preceding one. It can also appear as a brightness increase as the leading edge of the two CMEs interact, as is the case for the ejections from June 11, 1998 (see Figure 1). However, often, the only indication that multiple CMEs interacted on their way to Earth is when multiple Earth-directed ejections are observed by LASCO and a single structure (multiple-magnetic cloud events or complex ejecta) is observed at Earth.

It is the goal of this work to propose other evidence of CMEs interaction based on three-dimensional (3D) magneto-hydrodynamic (MHD) simulations, relying both on existing (Wind, ACE, STEREO) and future missions (LWS Sentinels, Solar Orbiter). We briefly summarize the simulations used for this study in Section 2, In Section 3, we discuss in situ synthetic measurements at $1 \mathrm{AU}$, followed, in Section 4. by a presentation of the possible in situ observations closer to the Sun by future missions. In Section 5, we examine possible white-light observations of interacting CMEs by the STEREO Heliospheric Imagers. In Section 6, we conclude and discuss other possible observational evidence of interacting CMEs not included in the present work.

\section{Simulations of the Propagation and Interaction of Multiple CMEs Between the Sun and the Earth}

The two simulations on which this study is based have been published in Lugaz et al. (2005) and Lugaz et al. (2007). Both simulations are performed with a 3-D MHD code (BATS-R-US). In Lugaz et al. (2005) (therafter Simulation 1), two identical out-of-equilibrium Gibson-Low magnetic flux ropes (Gibson and Low, 1998) are added 10 hours after each other onto the solar surface into a solar wind characteristic of solar minimum (see also Manchester et al., 2004, for a description of the models). The interaction of those two ejections results in the passage of a multiple-magnetic cloud event at Earth. The two magnetic sub-clouds are preceded by a single shock wave, the result of the merging of the two shock waves driven by the ejections. In Lugaz et al. (2007) (thereafter Simulation 2), we investigate three homologous eruptions from NOAA active region 9236 in November 24, 2000. The three ejections were separated by 10 and 6.5 hours respectively and of equivalent velocities (1000-1200 $\left.\mathrm{km} \mathrm{s}^{-1}\right)$. We use the solar wind model developed by Roussev et al. (2003) incorporating MDI magnetogram data and, which reproduces observations by Wind for the pre-eruption solar wind. We use out-of-equilibrium semi-circular magnetic flux ropes to initiate the three eruptions and are able to reproduce most of the LASCO and Wind observations. Simulation 2 was performed with the Space Weather Modeling Framework (for a description of the SWMF, see Tóth et al., 2005).

\section{Synthetic Observations at 1 AU}

Here, we compare the results at $1 \mathrm{AU}$ of Simulation 1 (solid line, thereafter referred as the interacting case) to the results of an identical but iso- 
lated CME (dash-dotted line from Manchester et al. (2004), thereafter referred as the isolated case), as seen in the left panel of Figure2. The goal is to find the differences at Earth between the simple superposition of two magnetic clouds and the result of the interaction of those two clouds.

In the interacting case, the single shock reaches 1 $\mathrm{AU}$ at time $\mathrm{t}=66$ hours, 6 hours before the shock in the isolated case. In order to directly compare the plasma parameters at $1 \mathrm{AU}$, the results shown on the left panel of Figure 2 are shifted in time, so that the arrival time of the shocks coincide.

In the interacting case, after increasing by a factor of 3.6 across the shock, the density decreases at first in the sheath, but then increases again before the contact discontinuity at the interface with the first magnetic cloud. This increase in density in the rear part of the sheath is specific to the interacting case. This is because the rear part of the sheath has been shocked twice before the shocks' merging, whereas the front part is formed after their merging. Thus, the rear part of the sheath has been compressed by a factor greater than $(\gamma+1) /(\gamma-1)=4$. However, the evolution of the density at the shock and in the sheath do not provide clear evidence of the merging of two shocks. Specifically in the interacting case, the maximum temperature is reached just behind the shock and not inside the magnetic cloud. This temperature jump at the shock is a factor of 2.5 larger than the one found for the isolated case. This larger temperature is associated with the process of shocks' merging. Since the density and velocity jumps are found to be similar between isolated and interacting cases, the temperature is the only clear indicator that the shock observed at Earth is the result of the merging of two shocks. Also, the larger density in the sheath is expected to have important consequences for the geo-effectiveness of the event (Farrugia et al., 2006).

One indication that the first magnetic cloud has been compressed and heated by a shock is that this cloud is hotter than the second one with a maximum temperature about $25 \%$ larger than that of the second cloud (and than that of the isolated cloud). However, this difference in temperature is smaller than the variation in temperature from one isolated magnetic cloud to the other. At Earth, the global structure composed by the two magnetic clouds is about $50 R_{\odot}$ wide and the second magnetic cloud accounts for about $80 \%$ of this width. This structure is fairly different from the simple superposition of two identical magnetic clouds. Also, the maximum magnetic field strength in the first cloud for the interacting case is only slightly larger (26 nT instead of $25 \mathrm{nT}$ ) than that of the isolated cloud. However, the maximum southward magnetic field in the first cloud reaches a maximum value of $-24.7 \mathrm{nT}(-20$ $\mathrm{nT}$ in the isolated case). The maximum southward magnetic field of the second cloud is very similar to the one from the undisturbed case. One can expect the geo-effectiveness of the interacting event to be larger, especially because the maximum southward $B_{z}$ is larger in the first cloud compared to the isolated case.

The reconnection region between the two magnetic clouds is associated with values of $\beta$ above unity, corresponding to the low magnetic field and high plasma temperature. This region is associated with the reconnection of the two clouds, which results in magnetic energy being transformed into thermal energy. Such high- $\beta$ interaction regions are described by Wang et al. (2003) for three different multiple-MC events in March-April 2001.

\section{Synthetic Observations Closer to the Sun}

There has been no systematic in-situ observations of solar transients between the Sun and the L1 point since the days of Helios and IMP (1980s).

The Living With a Star (LWS) Sentinels is a project of four spacecraft to be launched in the second half of the 2010s into elliptical orbits between 0.3 and $0.8 \mathrm{AU}$. Solar Orbiter is planed to be launched by the mid-2010s into an elliptical orbit around the Sun reaching distance as close as $45 R_{\odot}$ from the Sun. This will provide unique opportunities to study shocks propagation and interaction with preceding ejecta. $A C E$, Wind and the two STEREO spacecraft will provide complementary in-situ observations near Earth's orbit. Observations of shocks propagating into magnetic clouds are more likely to be made closer to the Sun than Earth's orbit. Near Earth, it is more likely to observe a shock having exited the magnetic cloud it overtook, as is the case for the March 20, 2003 event (Berdichevsky et al., 2005). An example of observations closer to the Sun is the complex stream observed by Helios 2 in April, 2-6, 1979 as reported by Farrugia \& Berdichevsky (2004), where two shocks appear to propagate inside preceding ejecta.

As an example of in-situ measurements close to the Sun, we consider the plasma properties of the two ejections from Simulation 1 as they would have 

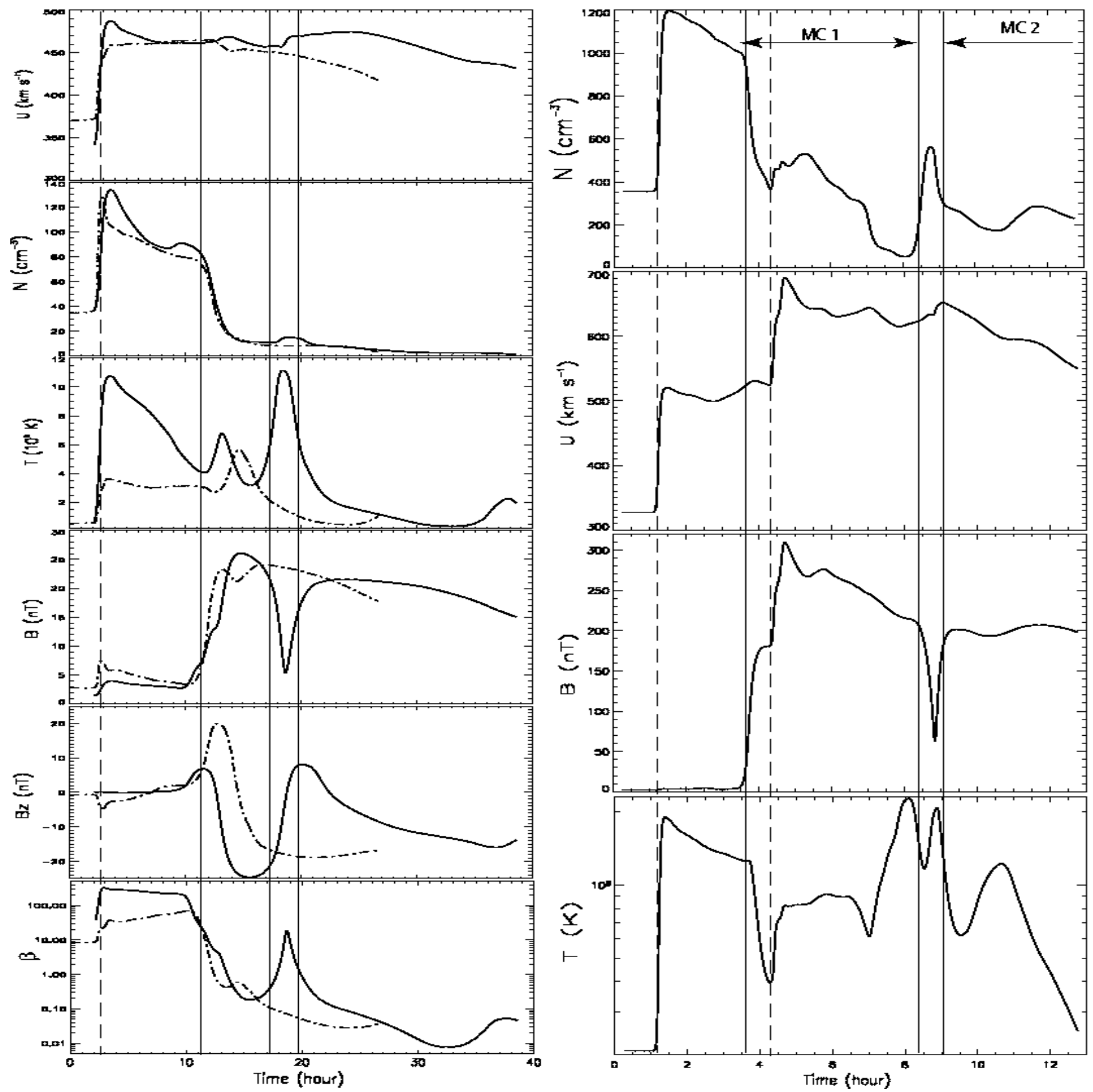

Fig. 2. Left panel: Solid line: Plasma properties near 1 AU for the two interacting CMEs. Dash-dotted line: Plasma properties near $1 \mathrm{AU}$ for the simulation by Manchester et al. (2004) of an identical isolated CME. The results are shifted in time in order to align the arrival of the shock fronts.

Right panel: Plasma properties near 0.33 AU for the two interacting CMEs. In both panels, the solid vertical lines show approximately the boundary of the two magnetic clouds, and the dash vertical line(s) show the position of the forward shock(s).

been observed by a satellite along the Sun-Earth line at a distance of $0.33 \mathrm{AU}$ from the Sun (see right panel of Figure 2). The results at $1 \mathrm{AU}$ are shown with the solid line on the left panel of Figure 2, At $0.33 \mathrm{AU}$, the trailing shock is in the front part of the leading cloud. Across the shock, the compression in density is about 1.32, the jump in the magnetic field strength is about 1.7, reflecting the modifications of the trailing shock properties by the extreme upstream conditions encountered (see Lugaz et al., 

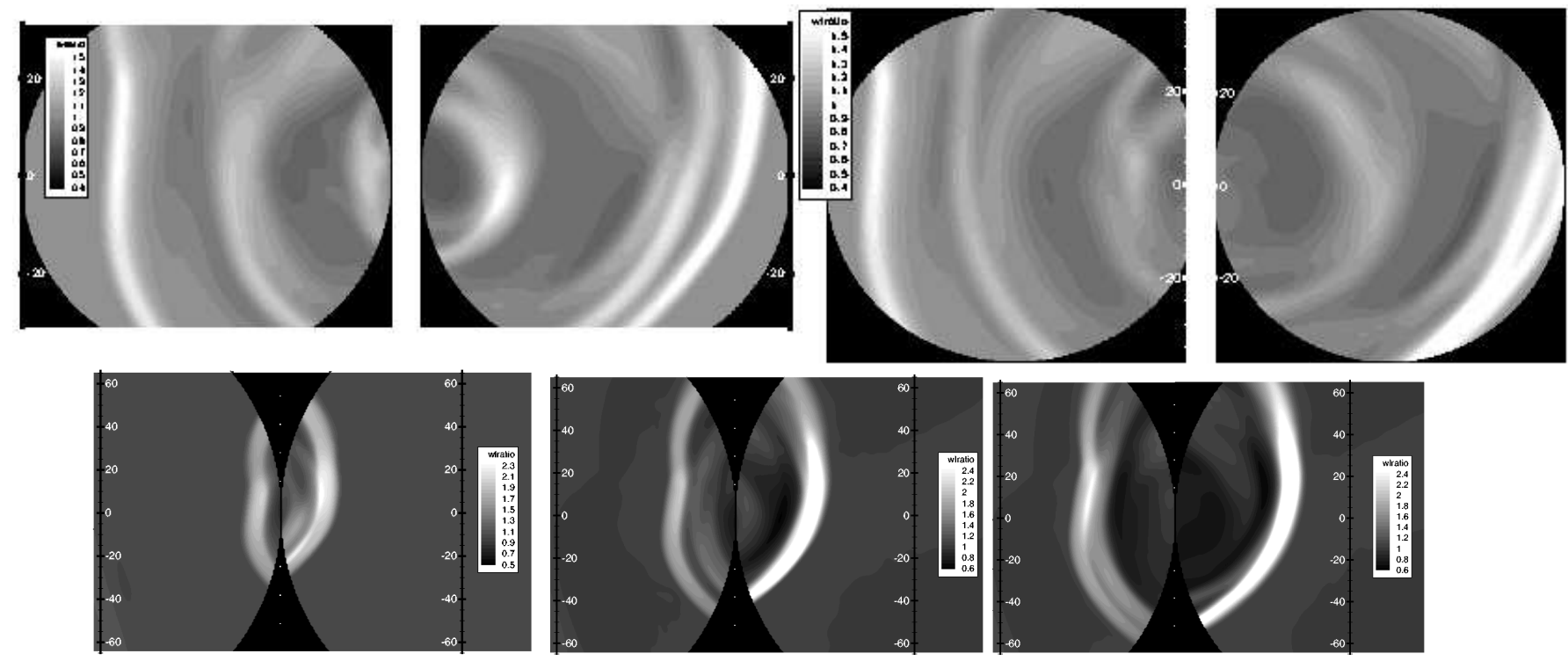

Fig. 3. Synthetic STEREO Heliographic Imagers observations of the interaction of 3 CMEs. Running ratio of consecutive images. Top: Heliospheric Imagers 1, 22 and 25 hours after the initiation of the first CME (1-hour cadence). Bottom: Heliospheric Imagers 2, 22, 28 and 34 hours after the initiation of the first CME (3-hour cadence).

2005, section 4, for more details). This is a case of shock propagating inside a magnetic cloud, also studied, for example, by Xiong et al. (2006).

The speed profile in the ejecta is not as uniform as observed at Earth, because the trailing shock is still propagating inside the first cloud and is still accelerating it. The temperature profile is also significantly different from that observed at $1 \mathrm{AU}$, with a much more homogenous sheath. Indeed, the larger decrease in temperature in the sheath observed at Earth is due to the sheath being made of a "new" sheath (shocked by the new shock) and an "old" sheath (twice shocked). Having spacecraft closer to the Sun than 1 AU will provide evolutionary evidence of CMEs interaction. Here, having both observations at 0.33 and $1 \mathrm{AU}$, we can conclude that the trailing shock propagates inside the cloud and, consequently, the single shock observed at $1 \mathrm{AU}$ is the result of the merging of multiple shocks. Also the part of the first cloud just downstream of the trailing shock has already the same speed as that of the second cloud, whereas the part of the first cloud not yet shocked is slower. We can conclude from this observation that the trailing shock is primarily responsible for the acceleration of the first cloud. Consequently, the uniform speed at $1 \mathrm{AU}$ is not due to momentum transfer between the clouds, but it is due to the acceleration of the cloud by the trailing shock and the presence of the second cloud which prevents the first one to decelerate after the shock's passage.
This conclusion can only be reached by having in situ observations of the trailing shock propagating inside the first magnetic cloud, as well as observations after the interaction between the ejections took place.

\section{Coronagraphic Observations by STEREO}

The STEREO mission has been successfully launched in October 2006. In this section, we consider synthetic observations by the Heliospheric Imagers 1 and 2 (HI1 and HI2) instruments onboard the two satellites. The HI1 instruments have a field-of-view of $20^{\circ}$ with an angular offset of $13.65^{\circ}$ towards the Sun-Earth line and the HI2 instruments have a field-of-view of $69^{\circ}$ with an angular offset of $53.35^{\circ}$ towards the Sun-Earth line. Figure 3 shows different synthetic white-light images based on Simulation 2 corresponding to the satellites' configuration one year after the launch (two spacecraft separated by $45^{\circ}$ ).

The top panels of Figure 3 show synthetic images similar to what $H I 1$ would have observed. Here, at time 22 hours, the three leading edges can be observed and distinguished in both HI1's fields-of-view at the same time. The 3-D structure of the CMEs is clearly visible. On the top right panel, the two leading edges have started interacting in HI1-B's field-ofview. This interaction is associated with an increase 
in the brightness, as the second shock propagating into the first dense sheath leads to a very large density increase. This is similar to what was observed in June 11, 1998 by LASCO C2 (see Figure 1).

The bottom panels of Figure 3 show synthetic images similar to what HI2 would have observed at three different times. The top and bottom left panels show the ejections at the same time in HI1 and HI2 fields-of-view. The brightness enhancement at the back of the first leading edge in the HI2-B is due to the second shock propagating into the leading edge of the first ejecta. Because HI1 and HI2 have different viewing angles, this feature is not yet observable in the HI1 plots. The middle bottom panel shows the effect of the different propagation speeds in the different quadrant of the heliosphere, as HI2-B observes the large brightness increase associated with the interaction of the first two ejections, whereas HI2-A still observes two distinct ejecta. Six hours later (right panel), the interaction region between the two shocks is visible in HI2- $A$ as well.

\section{Conclusion and Discussion}

We presented observational evidence of the interaction of multiple CMEs between the Sun and the Earth based on two 3-D MHD simulations. The main indicators of the interaction between multiple CMEs are the large temperature jump at the front shock and in the sheath and the high plasma $\beta$ in the region between the two clouds. The first feature indicates that the shock in front of the multiplemagnetic cloud event is the result of the merging of two shocks. The second feature indicates that reconnection happened between the two clouds. The short duration of the first cloud is also an indication that it has been compressed by an overtaking shock and that the natural tendency of the cloud to expand has been prevented by the presence of another cloud at its rear.

Having spacecraft dedicated to the study of space weather in orbits close to the Sun will provide additional evidence of shocks propagating inside magnetic clouds and of shocks' merging. Furthermore, they could help validate and test numerical models. Also, we predict that the Heliospheric Imagers onboard STEREO will provide coronagraphic images of the interaction of leading edges of CMEs propagating towards Earth. This interaction is associated with a brightness increase and CME "cannibalism", similar to what is observed by LASCO. Here we do not present synthetic LASCO observations, but the results found for the STEREO Heliospheric Imagers are directly applicable to LASCO. CME "cannibalism" and shock-shock interaction in the field-of-view of LASCO C3 have also been numerically investigated by Schmidt \& Cargill (2004). Also not presented here are synthetic in situ measurements at $1 \mathrm{AU}$ but at different angles from the Sun-Earth line, as STEREO-A and B will provide. Because of the 3 -D nature of CMEs and their shocks, STEREO A and B, ACE and Wind will be able to observe different phases of the interaction of multiple CMEs, which will also be very useful to validate and test $3-\mathrm{D}$ numerical models.

Acknowledgments The research for this manuscript was supported by Department of Defense MURI grant F004449 and ITR grant 0325332 at the University of Michigan. We thank the referees for useful comments. $\mathrm{SOHO}$ is a project of international cooperation between ESA and NASA.

W. M. has been partially supported by the grants NASA-NNX06AC36G and LWS03-0130-0149 in subcontract from George Mason. I. R. has been partially supported by the NSF grant ATM-0631790 (SHINE) and the Bulgarian NSF grant VU-01/06. N. L. would like to thank NSF for travel grants to the ISROSES meeting.

References

Berdichevsky, D. B., et al., Solar-HeliosphericMagnetospheric Observations on March 23-April 26, 2001: Similarities to Observations in April 1979, AIP Conf. Proc. 679: Solar Wind Ten, 679, 758,2003

Berdichevsky, D. B., Richardson, I. G., Lepping, R. P., Martin, S. F., On the origin and configuration of the 20 March 2003 interplanetary shock and magnetic cloud at 1 AU, J. Geophys. Res., 110, 9105, 2005

Burlaga, L. F., Plunkett, S. P., St. Cyr, O. C., Successive CMEs and complex ejecta, J. Geophys. Res., 107, 1, 2002

Farrugia, C., \& Berdichevsky, D., Evolutionary signatures in complex ejecta and their driven shocks, Ann. Geophys., 22, 3679-3698, 2004 
Farrugia, C. J., Jordanova, V. K., Thomsen, M. F.,Lu, G., Cowley, S. W. H., Ogilvie, K. W., A two-ejecta event associated with a two-step geomagnetic storm, J. Geophys. Res., 111, A11104, 2006

Gibson, S., \& Low, B. C., A time-dependent threedimensional magnetohydrodynamic model of the coronal mass ejection, ApJ, 493, 460, 1998

Gonzalez-Esparza, A., Santillán, A., Ferrer, J., A numerical study of the interaction between two ejecta in the interplanetary medium: one- and two-dimensional hydrodynamic simulations, Annales Geophysicae, 22, 3741, 2004

Gopalswamy, N., Yashiro, S., Kaiser, M. L., Howard, R. A., Bougeret, J. L., Radio signatures of Coronal Mass Ejection interaction: Coronal Mass Ejection cannibalism?, Astrophys. J. Lett., 548, L9194, 2001

Gopalswamy, N., A global picture of CMEs in the inner heliosphere, ASSL Vol. 317: The Sun and the Heliosphere as an Integrated System, eds. G. Poletto and S. T. Suess, 2004

Lugaz, N., Manchester, W. B., IV, Gombosi, T. I. , Numerical simulation of the interaction of two Coronal Mass Ejections from Sun to Earth, Astrophys. J., 634, 651-662, 2005

Lugaz, N., Manchester, W. B., IV, Roussev, I. I., Tóth, G., Gombosi, T. I. , Numerical investigation of the homologous CME events from active region 9236, Astrophys. J., 2007, accepted

Manchester, W. B., IV, Gombosi, T. I., Roussev, I., Ridley, A. J., DeZeeuw, D. L., Sokolov, I. V., Powell, K. G., Tóth, G., Modeling a space weather event from the Sun to the Earth: CME generation and interplanetary propagation, J. Geophys. Res., 109, 2017, 2004

Odstrčil, D., Vandas, M., Pizzo, V. J., MacNeice, P., Numerical simulation of interacting magnetic flux ropes, AIP Conf. Proc. 679: Solar Wind Ten, 679, 699-702, 2003

Reiner, M. J., Vourlidas, A., Cyr, O. C. S., Burkepile, J. T., Howard, R. A., Kaiser, M. L., Prestage, N. P., Bougeret, J.-L., Constraints on Coronal Mass Ejection dynamics from simultaneous radio and white-light observations, Astrophys. J., 590, 533, 2003

Roussev, I. I., Gombosi, T. I., Sokolov, I. V., Velli, M., Manchester, W., IV, DeZeeuw, D. L., Liewer, P., Tóth, G. Luhmann, J., A three-dimensional model of the solar wind incorporating solar magnetogram observations, Astrophys. J. Lett., 595, L57-L61, 2003
Schmidt, J. \& Cargill, P., A numerical study of two interacting coronal mass ejections, Ann. Geophys., 22, 2245-2254, 2004

Tóth, G., et al., Space Weather Modeling Framework: A new tool for the space science community, J. Geophys. Res., 110, 12226, 2005

Vandas, M., Fischer, S., Dryer, M., Smith, Z., Detman, T., Geranios, A., MHD simulation of an interaction of a shock wave with a magnetic cloud, J. Geophys. Res., 102, 22295-22300, 1997

Wang, Y. M., Ye, P. Z., Wang, S., Multiple magnetic clouds: Several examples during MarchApril 2001, J. Geophys. Res., 108, 6, 2003

Xiong, M., Zheng, H., Wang, Y., Wang, S., Magnetohydrodynamic simulation of the interaction between interplanetary strong shock and magnetic cloud and its consequent geoeffectiveness: 2 . Oblique collision, J. Geophys. Res., 111, 11102, 2006 